\title{
CAMBIO HISTÓRICO Y FORMA NARRATIVA EN «THE GOLDEN NOTEBOOK», DE DORIS LESSING
}

\author{
Pilar hidalgo andreu \\ Universidad de Málaga
}

D. H. Lawrence llamó a la novela «el encendido libro de la vida», y desde los comienzos del género la relación entre novela inglesa $e$ historia ha sido estrecha y significativa. En la primera gran década de la narrativa inglesa, los años que van desde Pamela (1740) a Tom Jones (1749), el ascenso social y cultural de la burguesía y su cambiante relación con la aristocracia queda simbolizado en el matrimonio de Pamela y Mr. B. ${ }^{1}$. La última década del siglo XvirI presenció el nacimiento primero de la novela jacobina y más tarde de la antijacobina, sin olvidar la ideología conservadora que subyace en el tono neutro de la narrativa de Jane Austen. Entre 1847 y 1848 la novela inglesa experimentó un cambio formal y temático que no podemos separar de la realidad de una de las décadas más turbulentas de la historia de Inglaterra, «los hambrientos años cuarenta"; tras la sensibilidad a veces exacerbada de Wuthering Heights, Jane Eyre, Mary Barton y Dombey and Son está el inmenso sufrimiento humano que acompañó al proceso de industrialización inglés.

Si el pensamiento de Darwin está tras la figura de Tess de los d'Urbervilles, y el de Freud tras diversas creaciones de la novela modernista; si la narrativa de los años treinta refleja la crisis económica y política de la época, mientras que la novela inglesa de la década de los cincuenta es producto del conformismo impuesto por la guerra fría, creo que, transcurridos más de veinte años desde la publicación de The Golden Notebook en 1962, disponemos ya de la

1 Terry Eagleton ofrece un tratamiento lúcido de este aspecto de Pamela en The Rape of Clarissa, Oxford: Basil Blackwell, 1982. 
perspectiva necesaria para afirmar que la aparición de la obra de Doris Lessing abrió una etapa en la novela inglesa que no ha concluido todavía. Si volvemos a los ejemplos anteriores, vemos que lo que definía a Tess era su indefensión ante fuerzas naturales y económicas; el héroe arquetípico de la literatura militante de los treinta se caracterizaba por su capacidad de compromiso y acción, y el de la nueva picaresca de los cincuenta por su marginalidad y pasividad. De manera algo semejante, hay dos aspectos de The Golden Notebook que resultarán proféticos de la evolución posterior de la novela inglesa: el carácter fragmentario de la técnica narrativa y el que la protagonista, Anna Wulf, sea una novelista que se muestra incapaz de seguir escribiendo. Me parece evidente que dos notas esenciales de la narrativa inglesa de los años sesenta y setenta han sido la experimentación formal y el cuestionamiento del propio status del género, si bien en Doris Lessing, y especialmente en The Golden Notebook, tanto la experimentación como el cuestionamiento son consecuencia de una crisis ideológica, y no, como será el caso de la mayoría de los autores posteriores, el producto de un interés teórico o parte de un juego retórico.

En el Prefacio que Doris Lessing escribió para una reimpresión de The Golden Notebook en 1971, aparece una observación que, además de ser profética, escrita como está dos años antes del inicio de la crisis económica mundial, viene a apoyar lo que he dicho más arriba. Lessing comenta el hecho de que en la época de publicación de la novela uno de los aspectos que más llamaron la atención de la crítica fue el elemento feminista de The Golden Notebook ${ }^{2}, \mathrm{y}$, tras declarar su apoyo a las reivindicaciones feministas, pasa a afirmar algo que resulta bastante original para la época en que fue escrito: \&I don't think that Women's Liberation will change much, though- not because there is anything wrong with their aims, but because it is already clear that the whole world is being shaken into a new pattern by the cataclysm we are living through: probably by the time we are through, if we do get through at all, the aims of Women's Liberation will look very small and

2 Conviene tener en cuenta que The Golden Notebook se anticipb al despertar feminista que se produjo en Estados Unidos a finales de los sesenta y en Europa poco más tarde. El punto de partida del nuevo feminismo, The Feminine Mysti$q u e$, de Betty Friedan, apareció en los Estados Unidos en 1963, y pasarían algunos años hasta que el feminismo de las obras polémicas y doctrinales se encarnara también en obras narrativas. El tema feminista (tomando el adjetivo en un sentido amplio) tiene una larga historia en la novela inglesa. Para un estudio de una de sus manifestaciones más interesantes, véase Boumel HA, Penny: "Women and the New Fiction 1880-1890m, en su Thomas Hardy and Women, Brighton: Harvester Press, 1982, 63-97. 
quaint ${ }^{3}$. Es indudable que entre los cataclismos a que se refiere Lessing, la existencia de arsenales nucleares capaces de destruir a la especie humana adquiere importancia capital en su imaginación; la larga secuencia novelística Children of Violence, comenzada en 1952 con Martha Quest, concluye con el holocausto atómico en The FourGated City (1969), y la guerra nuclear como culminación de la larga noche de la historia humana figura también en Shikasta (1979). En una entrevista concedida en 1969, Lessing relacionaba la sensación de ruptura y disolución en las relaciones personales con la experiencia de Hiroshima, y The Golden Notebook incluía el terror nuclear en su compleja malla de referencias.

Una primera aproximación a una novela tan extensa y prolija como The Golden Notebook puede partir de dos palabras: forma (Lessing emplea shape y pattern indistintamente) y caos. El problema de dar forma a la experiencia, como el problema de dar coherencia a la existencia, es un motivo constante en The Golden Notebook y constituye el mismo origen de su estructura narrativa. La novela está compuesta de una narración titulada Free Women (que la autora califica de aesqueleto» o «marco») y de los contenidos de cuatro cuadernos propiedad de Anna Wulf, la protagonista de Free Women. Los cuadernos (negro, rojo, amarillo y azul, respectivamente) y Free Women se entrecruzan varias veces hasta concluir en un breve cuaderno dorado final y volver a Free Women en la conclusión de la novela. Los modos narrativos son variados: narración tradicional en tercera persona, diarios, ensayo, parodia, «relatos dentro del relaton, material documental, etc.; sin embargo, el carácter metanovelístico de The Golden Notebook no depende tanto de la ruptura del ritmo narrativo (recurso, por lo demás, nada nuevo), sino de la manera en que la novela incorpora su propia crítica y deja al descubierto su condición ficticia. El cuaderno negro recoge la experiencia africana de Anna Wulf (muy semejante en contenido a A Ripple from the Storm y Landlocked), experiencia en la que bas 6 su primera y única novela Frontiers of War. Este cuaderno recoge también los diversos intentos de adaptar para el cine y la televisión la novela de Anna, situaciones que darán origen a ejercicios paródicos. La búsqueda de Anna Wulf de una forma narrativa parte de la relación entre novela y sociedad:

The novel has become a function of the fragmented society, the fragmented consciousness. Human beings are so divided, are becoming more and more divided, and more subdivided in

3 Lessing, Doris: «Preface», The Golden Notebook, Londres: Panther Books, 1973 (1962), 8-9. Todas las notas hacen referencia a esta edición y van entre paréntesis en el texto. La cursiva es mía. 
themselves, reflecting the world, that they reach out desperately, not knowing they do it, for information about other groups inside their own country, let alone about groups in other countries. It is a blind grasping out for their own wholeness, and the novel-report is a means towards it... Yet I am incapable of writing the only kind of novel which interests me: a book powered with an intellectual or moral passion strong enough to create order, to create a new way of looking at life.

( $G N, 79,80$. La cursiva es de Lessing.)

La dicotomía forma/caos aparece pronto en The Golden Notebook dentro de un diálogo entre Anna Wulf y su amiga Molly:

'What's in those diaries then?'

'They aren't diaries'

'Whatever they are'

'Chaos, that's the point'

$(G N, 60$.

El caos ideológico y personal en el que Anna Wulf se desenvuelve se traduce en la fragmentación (otra palabra clave en la novela). La consecuencia más obvia de la fragmentación es el hecho de que Anna recoja en cuadernos diferentes los diversos aspectos de su experiencia. Si el cuaderno negro se ocupaba de todo lo relacionado con Frontiers of War y los años pasados en Africa, el cuaderno rojo detalla la historia política de Anna, el cuaderno amarillo contiene una novela corta titulada The Shadow of the Third (que es a su vez una proyección de lo que sucede en la historia «principal», Free Women) y, finalmente, el cuaderno azul reproduce el diario que Anna ha llevado durante varios años. Además de la fragmentación física de la novela, hay un intento consciente por parte de Anna de mantener la separatividad de la experiencia y de no incluir en un cuaderno algo que pueda pertenecer a otro. Así, por ejemplo, encontramos en el cuaderno negro:

Because the experience with Mother Sugar and the experiments with the notebooks has sharpened my objectivity to the point where-but this kind of observation belongs to the blue notebook, not this one.

$(G N, 148$.

Igualmente, dentro de los esquemas para relatos que el cuaderno amarillo contiene aparece la siguiente observación: "(This sort of comment belongs to the blue notebook, I must keep them separate) $(G N, 520)$. Hay que esperar hasta el cuaderno dorado al final 
de la novela para encontrar mezcladas las diferentes experiencias de que se han nutrido los cuatro cuadernos; de este modo el cuaderno dorado reproduce verbatim una larga parrafada de Paul Tanner (personaje de The Shadow of the Third) que habia aparecido ya en el cuaderno amarillo. Es decir, el cuaderno dorado supone un intento deliberado de romper las barreras entre las diferentes áreas de experiencia marcadas por los cuadernos negro, rojo, amarillo y azul.

La estructura narrativa de The Golden Notebook, como la de cualquier novela, refleja la concepción del mundo de su autora en un momento determinado; un momento de crisis en el que la experiencia aparece fragmentada. Pero a pesar de esa fragmentación en la que The Golden Notebook insiste de una manera tan gráfica, y a pesar de las referencias a "la crisis de la novela», "el debate sobre la novela", etc., el texto no cuestiona la capacidad de la novela de reflejar la realidad y transmitir significados. Por el contrario, si algo caracteriza a The Golden Notebook es la acumulación de material mimético, de detalles sobre infinidad de ambientes, personajes y hablas: entornos naturales y urbanos, comidas y bebidas, aspecto externo de seres humanos, ropas, decoración de interiores, jergas políticas y críticas, recortes de prensa, etc., sin olvidar la presentación minuciosa de la interioridad. La reproducción de la realidad es todavía importante para Lessing en The Golden Notebook, y ya en las primeras páginas del cuaderno negro Anna Wulf reflexiona sobre la posibilidad de describir a un personaje a través de una serie de adjetivos del tipo implacable/bondadoso, frio/cariñoso, sentimental/realista: «But really what I discovered, though I didn't know it then, was that in describing any personality all these words are meaningless... once I say that words like good/bad, strong/weak, are irrelevant, I am accepting amorality, and I do accept it the moment I start to write 'a story', 'a novel', because I simply don't care. All I care about is that I should describe Willi and Maryrose so that a reader can feel their reality» $(G N, 88,89$. La cursiva es mía).

En The Golden Notebook la crisis del realismo es una cuestión de cómo, no de por qué; la relación entre literatura y vida a través del lenguaje no se cuestiona. Una de las reflexiones de Anna en el cuaderno azul establece la conexión entre ambas de manera automática, y señala además la primacía de la conciencia y la sensibilidad del individuo dentro del proceso creador. Igual que Ulysses o

4 Remito al lector interesado en las últimas aportaciones teóricas sobre el tema a los trabajos incluidos en «Fictionality and Reference», Poetics Today, Volume 4, Number 1 (1983). 
Mrs. Dalloway, The Golden Notebook no renuncia al mundo fenomenológico, sino que debate abiertamente los problemas de la representación ${ }^{4}$. El énfasis en la forma es insistente:

'... tell me why some books are for the minority'

I thought, and then said: 'It's a question of form'

'Form? What about the content of yours? I understand that you people insisted on separating form and content?'

'My people may separate them, I don't. At least, not till this moment. But now I'll say it's a question of form. People don't mind immoral messages... And they like messages saying that murder is bad, cruelty is bad, and love is love is love is love. What they can't stand is to be told it all doesn't matter, they can't stand formlessness.'

$(G N, 461$.

La preocupación por los problemas formales de la narrativa está en el origen de uno de los episodios más interesantes de la novela. En septiembre de 1954 (el espacio temporal de The Golden Notebook va de 1944 a 1957) Anna Wulf anota en su diario (cuaderno azul) la decisión de describir minuciosamente todos los acontecimientos de un solo día. Dado que en The Golden Notebc ok las cuestiones formales nunca se separan de la vida políitca : afectiva de la protagonista, ese día que empieza como un experimento literario resulta ser decisivo para Anna: Michael, el hombre con el que ha mantenido una intensa relación amorosa desde hace cinco años, la abandona sin aviso, y la propia Anna interrumpe bruscamente su militancia en el partido comunista. Lo que subyace en el plan de Anna es el propósito de escapar al proceso mediador del escritor y dejar constanica de los incidentes según se van produciendo. Lógicamente, como sucedía en Ulysses, pasan a primer plano aspectos fisiológicos que la novela clásica había eludido; la famosa visita al retrete de Leopold Bloom tiene su equivalente en los detalles sobre la menstruación de Anna ${ }^{5}$. En conjunto, la presentación que Anna Wulf hace del 16 de septiembre de 1954 es una mezcla interesante de vida física e ideológica. Desde que se levanta hasta que llega a la oficina del partido, Anna está dominada por la biología y la cotidianeidad. Tiene el último encuentro sexual con Michael, despierta a su hija, prepara el desayuno, arregla la casa, compra los ingredientes que necesita para una cena especial con Michael y riega las macetas. A pesar de que el día resultará traumático para Anna, este episodio

5 Es interesante señalar que Anna se refiera a este episodio de Ulysses cuando reflexiona sobre el ejercicio literario que se ha propuesto para ese día. 
del cuaderno azul contiene uno de los pocos momentos de gozo en la tarea realizada y de plenitud afectiva en la obra de Lessing; habrá que esperar al mundo fantástico de The Marriages Between Zones Three, Four, and Five (1980) para encontrar algo parecido. Y es muy significativo el que los dos momentos de gozo de Anna Wulf se produzcan mientras lleva a cabo actividades tan tradicionalmente femeninas como cuidar de su hija y preparar una buena cena para compartir con el hombre a quien quiere.

La descripción detallada de este día de septiembre sirve también para que The Golden Notebook incorpore, aunque sea parcialmente, su autocrítica. Tras haber experimentado con una narrativa basada en la anotación precisa de datos a lo largo de más de treinta páginas, Anna reconoce el fracaso y se autocorrige: la segunda entrega del cuaderno azul concluye con una entrada en el diario de apenas nueve líneas que presenta en forma telegráfica los mismos acontecimientos que Anna acaba de describir tan detenidamente. Es decir, en The Golden Notebook Anna Wulf presenta las experiencias y emociones de los que se nutre su arte y al mismo tiempo el proceso de elaboración creadora (decisiones, supresiones, ampliaciones, cambios de énfasis) del que surgirá la novela, sin olvidar el elemento de autocrítica al que aludía más arriba ${ }^{6}$.

La parodia ha formado parte de la novela desde su orígenes. En la narrativa inglesa de los siglos XvIII y XIX los más conocidos títulos paródicos (Shamela, Northanger Abbey, Catherine, la parodia de la "silver-fork school" en Nicholas Nickleby) suelen aparecer al comienzo de la carrera de los autores, como si los escritores jóvenes sintieran la necesidad de realizar un rito de exorcismo respecto a sus predecesores o poner a prueba su capacidad de recreación ${ }^{7}$. Dentro de la novela contemporánea en Inglaterra, el caso de parodia que más se asemeja a los ejemplos antes citados es la segunda novela de David Lodge, The British Museum Is Falling Down (1965). The Golden Notebook incorpora la parodia, si bien en la novela de Lessing no cumple la función de exorcismo ni de punto de partida para un

6 No hay duda de que la inclusión de autocrítica en el texto hace correr el riesgo de una cierta complacencia. En mi opinión, Doris Lessing logra salvar el escollo; no así, por ejemplo, John Fowles, en The French Lieutenant's Woman (1969) y Mantissa (1982). Para un uso inteligente de la incorporación de la autocritica al texto (en este caso crítico), véase Bernard Sharratt, Reading Relations: Structures of Literary Production, Brighton: Harvester Press, 1982.

7 Valentine Cunningham incluye un texto paródico de Charlotte Brontë en el apéndice a su Everywhere Spoken Against (Dissent in the Victorian Novel), Oxford: Clarendon Press, 1975. El manuscrito está fechado en 1837, cuando la autora tenía veintiún años, lo cual confirma lo antes dicho sobre el momento paródico en la carrera de los novelistas victorianos. 
cuestionamiento del género (el caso, por ejemplo, de The French Lieutenant's Woman). La parodia en The Golden Notebook no tiene una función específica aparente, como no sea la de poner una nota divertida en una obra con frecuencia sombría. Si dejamos a un lado las parodias que no toman como punto de partida a otras obras originales (las «versiones» de Frontiers of War que Anna Wulf imagina, o la inclusión de distintas críticas soviéticas de la misma novela, los estilos parodiados en The Golden Notebook tienen poco que ver con la práctica de Doris Lessing. Tampoco se trata de parodias de autores u obras concretos; el objeto de la parodia es más bien un ambiente o una forma determinada de hacer las cosas. Así, en el diario de una escritora que Anna y un amigo americano inventan, existe un cierto aroma de las obras teatrales de Eliot (especialmente The Cocktail Party). La viñeta titulada "The Romantic Tough School of Writing» parodia lo que podríamos calificar de subproductos hemingwayanos, mezcla de falsa simplicidad linguística (la del Hemingway de los años veinte era auténtica, y el resultado de un intenso ejercicio estilístico) y brutalidad afectiva que tuvo cierto predicamento en los años treinta y cuarenta en Estados Unidos. Es decir, la parodia literaria de The Golden Notebook se dirige hacia modos ideológicos y artísticos que están muy alejados de los de Lessing. Otro dato interesante es el hecho de que los fragmentos paródicos estén cuidadosamente delimitados y no pasen a formar parte del texto central.

El carácter pionero de The Golden Notebook sólo se puede apreciar si situamos la novela en su contexto histórico ${ }^{8}$. La eclosión innovadora y radical que supuso el Modernismo en la novela de habla inglesa en los años veinte y el enriquecimiento temático de los años treinta fue seguido por el tono menor del género en los años cuarenta (con las inevitables excepciones: por citar una, el Modernismo tardío de Under the Volcano). Por lo que a la década de los cincuenta se refiere, resulta melancólico reflexionar en la infinidad de artículos, libros y tesis doctorales que se han escrito basados en la premisa de que a partir de 1953 y 1954 (fechas de publicación de Hurry On Down y de Lucky Jim y Under the Net, respectivamente) la novela inglesa inició un proceso de renovación importante. Si algo caracteriza a las obras de Amis y Wain es el conformismo y el provincialismo inseparables de un concepto trasnochado del realismo. Iris Murdoch es

8 Que este carácter no fue en modo alguno evidente en la época de publicación de la novela lo podemos comprobar revisando los comentarios críticos de entonces, y los estudios generales sobre narrativa contemporánea que aparecieron en los años sesenta. En una obra tan conocida e influyente como Tradition and Dream, de Walter Allen (publicada en 1964), encontramos la asombrosa afirmación de que el principal interés de The Golden Notebook es de tipo sociológico. 
un caso aparte, y no era necesaria ninguna perspectiva histórica para haberse dado cuenta de que su encasillamiento con los anteriores resultaba incongruente". Frente a la engañosa "renovación" de 1954, la publicación de The Golden Notebook ocho años más tarde marca el comienzo de una etapa durante la cual el realismo tradicional de la novela inglesa va a ser sometido a prueba, revisado, cuestionado $y$, en algunos casos, abandonado; en la que la novela va a reflejar las alteraciones en la relación entre conciencia individual e historia, y en la que la propia razón de ser del género va a ser puesta en tela de juicio. Sin embargo, un rasgo que separa la obra de Lessing de otras novelas en las que la crisis del realismo es también patente es la confianza que Lessing demuestra en el valor referencial de la literatura; concretamente, en la capacidad que posee la novela de reproducir la realidad, crear personajes y transmitir una visión moral. En palabras de Lessing, la experimentación formal de The Golden Notebook tenía como objetivo último el que la novela lograra "to talk through the way it was shaped" (Prefacio, GN 14). $Y$, naturalmente, la separatividad que la estructura narrativa de la novela deja al descubierto de manera tan llamativa nos habla de inseguridad, sufrimiento y caos.

Decia al principio que caos era una de las palabras claves en The Golden Notebook. Si tenemos en cuenta el papel que una guerra nuclear desempeñará en The Four-Gated City y Shikasta y los frecuentes pronunciamientos de la autora sobre el peligro atómico, es fácil llegar a la conclusión de que la parálisis de la voluntad que afecta a varios personajes de The Golden Notebook está relacionada con el riesgo de un aniquilamiento total. No es casual el que, cuando el diario de Anna Wulf en el cuaderno azul deja paso a una serie de recortes de periódicos, el primer recorte contenga información sobre la bomba de hidrógeno. Con todo, la crisis de la que surge The Golden Notebook tiene un origen ideológico más concreto; tan concreto, $\mathrm{y}$ además incorporado de una manera tan explícita al texto, que es sorprendente la persistencia con la que la crítica ha eludido este aspecto fundamental de la novela. Ello se debe a cierta falta de tradición política en la novela inglesa. En un país que creó la novela moderna en el XVIII, la convirtió en el género dominante en el XIX, y revolucionó las formas narrativas en el $\mathrm{xx}$ (además de crear sub: géneros como la novela gótica, la histórica, la utópica, la de aventuras, la de detectives, y la ciencia-ficción), la novela política no ha

9 Es fácil establecer una relación entre el quietismo de la novela inglesa en los años cincuenta y la situación sociopolítica del país. Cfr. Hewinson, Robert: In Anger (Culture in the Cold War, 1945-60), Londres: Weidenfeld and Nicolson, 1981, passim. 
sido cultivada por los grandes maestros. De ahí que Doris Lessing expresara su asombro ante la ceguera con la que los críticos acogieron The Golden Notebook, con una salvedad importante: "As for the rest-well, it is no accident that $I$ got intelligent criticism from people who were, or who had been, marxists. They saw what I was trying to do. This is because Marxism looks at things as a whole and in relation to each other- or tries to, but its limitations are not the point for the moment" 10 (Prefacio, GN, 14).

La experiencia de Anna Wulf en The Golden Notebook es arquetípica de la de miles de personas en Europa occidental durante la posguerra: el abandono, progresivo o brusco, del ideal revolucionario que había sostenido la lucha contra el fascismo. La fecha exacta varía según los casos; los procesos de Moscú antes del comienzo de la guerra habían sembrado ya la duda en los espíritus más lúcidos (algo que Angus Wilson reflejará en la experiencia de Quentin Matthews en No Laughing Matter). Para otros, entre ellos Orwell, la experiencia desencadenante fue la participación en la guerra civil española. Tras la invasión alemana de la Unión Soviética, el activismo comunista en Inglaterra gozó de un ambiente favorable que no había tenido nunca; por vez primera, los dos grandes objetivos de derrotar al nazismo y defender a ultranza a la Unión Soviética se reforzaban mutuamente. Doris Lessing había descrito ya en $A$ Ripple from the Storm (1958) los efectos de la alianza anglosoviética en un lugar tan alejado de los centros europeos de poder como Zambesia ${ }^{11}$. En realidad, la historia política de Anna Wulf tiene numerosos puntos de contacto con la de Martha Quest; es en cierto modo la de Martha Quest antes de marchar a Inglaterra, aunque The Four-Gated City desarrolle en una dirección distinta la experiencia de la auténtica Martha Quest a partir de su llegada a Londres. A Ripple from the Storm y Landlocked son la crónica de los años de compromiso total y actividad frenética, y Lessing tiene mucho que decir sobre la descarga de energía que supone la confianza en un cambio profundo

10 Para un ejemplo reciente de este tipo de lectura, véase McCRindue, Jean: «Reading The Golden Notebook in 1962n, en TAYLoR, Jenny (ed.): Notebooks/ Memoirs/Archives (Reading and Rereading Doris Lessing), Londres: Routledge and Kegan Paul, 1982, 43-56.

11 En su trilogía Sword of Honour sobre la segunda guerra mundial, Evelyn Waugh presenta el caso contrario, el del inglés conservador para quien la guerra tiene sentido mientras la Alemania nazi y la Rusia soviética están aliadas. Esta es la reacción de Guy Crouchback ante el pacto germanosoviético de 1940: *... now, splendidly, everything had become clear. The enemy at last was plain in view, huge and hateful, all disguise cast off. It as the Modern Age in arms. Whatever the outcome there was a place for him in that battle.» [Waugh, Evelyn: Men At Arms, Harmondsworth: Penguin Books, 1978 (1952), 12.] 
de la vida humana. La oleada de entusiasmo ha pasado ya al comienzo de The Golden Notebook. El mismo día en que decide abandonar el partido comunista inglés, Anna Wulf reflexiona: «... when I leave the Party, this is what I am going to miss-the company of people who have spent their lives in a certain kind of atmosphere, where it is taken for granted that their lives must be related to a central philosophy" ( $G N, 338)$. Como suele ser habitual en Lessing, el énfasis está puesto no en el funcionamiento de las instituciones, sino en los efectos sobre el individuo. Anna Wulf de nuevo: "The real crime of the British Communist Party is the number of marvellous people it has either broken or turned into dry-as-dust hair-splitting office men, living in a close group with other communists, and cut off from everything that goes on in their own country" $(G N, 339)$.

El tratamiento del año 1956 confirma la excepcionalidad de The Golden Notebook dentro de la novela inglesa. La breve guerra por el canal de Suez que terminó con la humillación de Gran Bretaña ha adquirido con el tiempo un profundo valor simbólico. La crisis de Suez supuso la demostración palpable de que Inglaterra se había convertido en una gran potencia de segunda clase incapaz de mantener una presencia global. Para la izquierda inglesa, la intervención en Egipto demostró la irresponsabilidad criminal de la clase dirigente; de ahí el papel que Suez desempeña, por ejemplo, en The Entertainer (1957) y en Plenty (1979), dos piezas teatrales muy distintas y con una distancia de más de treinta años entre sí. Sin embargo, en The Golden Notebook 1956 no es el año de Suez, sino el año del XX Congreso del P.C. U.S., y una vez más Doris Lessing da prueba de su certero instinto histórico. Aunque Ana Wulf se había marchado del partido dos años antes, el momento de la verdad para miles de personas no llegó hasta $1956^{12}$. Es fácil ver con cierto cinismo la reacción de incredulidad y horror de los comunistas occidentales ante las «revelaciones" sobre un estado de cosas que venia siendo denunciado desde hacía más de treinta años; es necesario hacer un gran esfuerzo de imaginación histórica (especialmente difícil en los años ochenta) para comprender el inmenso caudal de esperanza que había generado la revolución rusa, y el efecto devastador ante el derrumbamiento de una cierta concepción del futuro. Doris Lessing había mostrado ya la fuerza de la visión (y la capacidad de autoengaño del ser humano) en $A$ Ripple from the Storm, en el significativo episodio en el que Martha y Marjorie, descontentas con el papel asig-

12 Mil novecientos cincuenta y seis fue el año en que abandonó el partido comunista E. P. Thompson, una de las figuras más influyentes del marxismo inglés contemporáneo. 
nado a las mujeres en la sociedad colonial, dan rienda suelta a su fantasía:

For some time, they sopke of the lives of the women in the Soviet Union. They lapsed into silence, smiling, pursuing the same fantasy: They were in the Soviet Union. They walked into some factory or industry, which was run by a woman, who was their age, or perhaps a little older, someone competent, matterof-fact, sympathetic. There would be little need for talk even: a smile and a squeeze of the hand would be enough, for this woman would understand at once why they needed to be given work that would absorb the best of themselves, why they needed time for study... ${ }^{13}$

El tema político está relacionado con el problema de cómo dar forma adecuada a la narrativa y con lo que creo es el meollo emotivo de las varias capas de relatos en The Golden Notebook: el sufrimiento humano. La relación de Anna Wulf con Michael (que Ella y Paul repiten en la novela corta del cuaderno amarillo) está marcada por un sentimiento casi insoportable de pérdida y añoranza, y la infelicidad es el elemento común a los varios relatos dentro del relato, cartas, esquemas y narraciones secundarias que se suceden en la novela. Es interesante el que tanto Anna como Ella estén a cargo de una misma especie de consultorio sentimental: las cartas de militantes comunistas que Anna recibe en la editorial del partido y la correspondencia de que Ella se ocupa en una revista femenina constituyen un abrumador historial de frustraciones, soledad y desamor. El problema eterno de la presencia del mal en el mundo es el nexo entre las dispares historias personales de The Golden Notebook; perdida la fe en la interpretación marxista de la historia, las raíces del mal se intuyen como fuerzas oscuras que minan las aspiraciones humanas ${ }^{14}$ : "... a vision of some dark, impersonal destructive force that worked at the roots of life and that expressed itself in war and cruelty and violence" $(G N, 192)$.

Los recortes de periódico que Anna va pegando en el cuaderno azul son una crónica de violencia, terror e injusticia. Pero bajo esta exhibición del dolor universal aparece la insistente nota de la infelicidad femenina. La relación entre hombre y mujer es siempre insatisfactoria en The Golden Notebook. Unas veces se trata de inadecución sexual (Anna y Willy, Ella y su marido, Ella y el cirujano norteame-

13 Lessing, Doris: A Ripple from the Storm, Londres: Panther, 1976 (1958), 168.

14 Precisamente la mitología de Shikasta (1979) intentará dar una explicación a la presencia del mal en el mundo. 
ricano, Molly y el actor, Anna y Nelson); pero en las dos relaciones fundamentales de la novela (Anna y Michael, Ella y Paul) el fracaso es bastante más complejo. En lo que se ha convertido en uno de los pasajes más citados del libro, Anna Wulf interrumpe la historia de Ella y Paul en The Shadow of the Third con el siguiente comentario: "Sex. The difficulty of writing about sex, for women, is that sex is best when not thought about, not analysed. Women deliberately choose not to think about technical sex. They get irritated when men talk technically, it's out of self-preservation: they want to preserve the spontaneous emotion that is essential for their satisfaction" $(G N, 219)$. Tras contar un pequeño incidente que ejemplifica lo que acaba de decir, la voz en primera persona de Ann se convierte de nuevo $\in$ en el narrador de The Shadow of the Third y pasa a comentar la relación entre Ella y Paul en los siguientes términos:

... she immediately experienced orgasm. Vaginal orgasm that is. And she could not have experienced it if she had not loved him. It is the orgasm that is created by the man's need for a woman and his confidence in that need... A vaginal orgasm is emotion and nothing else, felt as emotion and expressed in sensations that are indistinguishable from emotion... there can be a thousand thrills, sensations, etc., but there is only one real female orgasm and that is when a man, from the whole of his need and desire takes a woman and wants all her response.

$(G N, 220$.

La nota de protesta femenina que suena tan insistentemente en The Golden Notebook no debe hacernos perder de vista las implicaciones profundas del texto. La historia tan irónicamente titulada Free Women se presta a una lectura muy negativa desde un punto de vista feminista, en tanto que la no aceptación de los roles convencionales desemboca en la soledad y la amargura, y falta también la compensación del trabajo (Anna Wulf está bloqueada como escritora, y Ella hace un trabajo que desprecia). Lessing ha rechazado la etiqueta feminista (como cualquier otra clase de etiqueta) ${ }^{15}$, y su posición independiente se manifiesta en las discrepancias que existen entre su tratamiento narrativo de aspectos claves de la ideología feminista. Tomando The Golden Notebook como texto básico, la afir-

15 Sin embargo, la tendencia general al encasillamiento, y la identificación Lessing/The Golden Notebook (unida al desconocimiento de la evolución de la escritora) ha dado origen a numerosas confusiones, como se pudo apreciar en la mesa redonda con Doris Lessing celebrada en la Universidad Autónoma de Madrid en 1983. 
mación rotunda de la superioridad del orgasmo vaginal y la presentación de la homosexualidad masculina (en la relación entre Ivor y Ronnie) están muy lejos de los postulados del feminismo radical. Lo que la novela si presenta es la peculiar fragmentación de la vida femenina. Anna incluye la experiencia en la anotación minuciosa que hace de un día cualquiera de su vida:

I realise that what I used to refer to, to Mother Sugar, as 'the housewife's disease' has taken hold of me. The tension in me, so that peace has already gone away from me, is because the current has been switched on: I must-dress-Janet-get-herbreakfast-send-her-off-to-school-get-Michael's-breakfast-don't-forgetI'm-out-of-tea-etc.-etc... With this useless but apparently unavoidable tension resentment is also switched on. Resentment against what? An unfairness. That I should have to spend so much of my time worrying over details. The resentment focuses itself on Michael; although I know with my intelligence it has nothing to do with Michael. And yet I do resent him, because he will spend his day served by secretaries, nurses, woven in all kinds of capacities, who will take this weight off him. (La cursiva es mía.)

$(G N, 329$.

The Golden Notebook incorpora también un aspecto esencial de la experiencia femenina como es la necesidad de asumir "papeles" que tiene poco que ver con la auténtica personalidad de una mujer, pero que responden a lo que la sociedad espera de ella. En Children of Vio'ence esta asunción de "papeles" resultaba en la creación que Martha Quest hacía de "Matty» y en el conflicto permanente entre lo que «Matty» representaba y la realidad última de Martha. El desdoblamiento psíquico de la mujer moderna es uno de los centros de interés de la literatura feminista, y está relacionado con el cuestionamiento y el rechazo del psicoanálisis y la psiquiatría clásicos. La importancia que lo que se solía llamar locura tiene en la literatura hecha por mujeres (aunque no necesariamente feminista) es evidente, y podemos relacionarla con la obligación de asumir papeles falsos y la consiguiente división interior ${ }^{16}$. La mujer que se derrumba, sufre depresiones, es incapaz de realizar los gestos de la vida cotidiana, es un personaje clave en Lessing, y la reacción social que representa el tratamiento psiquiátrico es el equivalente moderno de la antigua per-

16 El fenómeno no es exclusivamente contemporáneo, aunque, naturalmente, aparezca en el siglo xx con mayor frecuencia y libertad. Cfr. GILBERT, S. M., y Gubar, S.: The Madwoman in the Attic, New Haven: Yale Univversity Press, 1979, passim. 
secución por brujería ${ }^{17}$. El derrumbamiento psíquico suele ir acompañado de la huida del mundo exterior hacia un enclaustramiento en un espacio cerrado; de ahí la aparición constante en Lessing de la habitación como refugio y centro de experimentación con uno mismo ${ }^{18}$. Se trata de una habitación en la que predominan los colores básicos (blanco, negro y rojo en la de Anna en The Golden Notebook, blanco y negro en la de Jack en The Four-Gated City), y en ambas novelas, el mundo exterior, excluido mediante el enclaustramiento, está presente en la habitación en los recortes de periódicos pegados a las paredes (la habitación de Anna Wulf), o en los recortes, estadísticas, mapas, etc., que invaden progresivamente el estudio de Mark Coldrige en The Four-Gated City.

La gran variedad de personajes y situaciones en The Golden Notebook puede encubrir un dato capital: el que la escritura es la principal actividad de la novela. Evidentemente Anna Wulf es una escritora, y su novela Frontiers of War figura de forma destacada en el texto, pero es significativo el número de personajes que recurren a la escritura: desde los dirigentes del partido comunista inglés, cuya secreta vocación es la literatura, hasta los oscuros militantes de cuya correspondencia se encarga Anna y que sacrifican sus escasas horas de ocio a la producción de patéticas obras literarias, sin olvidar al viejo trotskista que ha pasado su vida acumulando materiales para escribir una historia "verdadera" de la revolución rusa, la novela sobre el suicidio que escribe Ella, los guiones cinematográficos y obras de teatro de Nelson, los poemas del padre de Ella, la novela sobre Argelia de Saul Green, el relato del camarada Ted, las parodias que componen Anna y James Schaffer, el melodrama francés en el que está interesada la revista de Ella, las cartas de los lectores, el artículo de Stalin que Anna y sus camaradas intentan analizar... Y si la escritura se halla en el centro de la novela, algo semejante ocurre con la interpretación. El texto ofrece diversas lecturas de Frontiers of War (la del productor de televisión, las de varios críticos soviéticos, la de la agente literaria norteamericana), así como las interpretaciones contradictorias que Ella y el doctor West ofrecen de las cartas de los lectores de la revista en la que los dos colaboran; la lectura que Paul hace de la novela de Ella,

17 No es casual el que Caryl Churchill explore la relación entre brujería y misoginia en la sociedad del XvII en su pieza teatral Vinegar Tom (1976).

18 Frederick R. Karl ha visto cierta similitud en la recurrencia del espacio cerrado en Lessing y en las primeras obras de Pinter. Cfr. «Doris Lessing in the Sixties: The New Anatomy of Melancholy", incluido en MEYER SPAcks Patricia (ed.): Contemporary Women Novelists, Englewood Cliffs, N. J.: PrenticeHall, 1977, 55-74. 
o Tommy de los cuadernos de Anna; las revistas soviéticas que Anna lee buscando entre líneas indicios sobre cambios en la política del partido; el diferente significado que Anna y Rose dan a la carta de la mujer de un militante, etc. Sin embargo, esta inmersión en la palabra escrita no supone todavía el abandono de la realidad, sino más bien la obsesión por captar una realidad psicológica, histórica y política extraordinariamente compleja. En énfasis en «lo real» es omnipresente en The Golden Notebook; ya he citado el propósito expresado por Anna en la primera parte del cuaderno negro de describir a Willi y Maryrose de tal manera que el lector pueda «sentir su realidad». En las relaciones entre hombres y mujeres, real es también la palabra clave; Anna y Molly encuentran cada vez más difícil conocer a "real men». La atracción de George Hounslow reside en que es uno de los pocos hombres que "really, very much, needed women". He citado también el pasaje en que Anna/Ella distingue entre "real orgasm" y otro tipo de experiencia sexual.

A pesar de su talante experimental, producto de la conciencia del cambio histórico, The Golden Notebook no llega a poner en duda la capacidad que posee la novela de representar la realidad, lo cual no impide que, como demuestra el episodio en que Anna narra dos veces lo sucedido un día de 1954, el texto sea consciente del proceso de transformación que impone el mismo hecho literario. La conciencia de las limitaciones de la novela en tanto que ficción queda también al descubierto en una conversación entre Anna Wulf y el hijo de Molly:

It's a different kind of sensibility. Don't you see? In a day when I buy food and cook it and look after Janet and work, there's a flash of madness-when I write it down it looks dramatic and awful. It's just because I write it down. But the real things that happened in that day were the ordinary things. (La cursiva es mía.)

$(G N, 272$.

The Golden Notebook planteaba abiertamente la inevitable conexión entre cambios en la experiencia y cambios en las estructuras narrativas, y lo hacía en una época en la que la nota dominante de la novela inglesa era el provincianismo estético e ideológico, y el rechazo de las innovaciones del Modernismo. El caso de Lessing es todavía más interesante si tenemos en cuenta que había comenzado su carrera en 1950 con The Grass Is Singing, modelo de narrativa tradicional, y había escrito en la década de los cincuenta los tres primeros títulos de la secuencia Children of Violence, continuando así la gran tradición del Bildungsroman. No es extraño que en 1955 
C. P. Snow, defensor a ultranza del retorno a los moldes narrativos clásicos, incluyera a Lessing dentro de una lista que escritores jóvenes que, según Snow, desdeñaban las posibilidades estéticas de la novela y aspiraban a reflejar sin mediaciones los problemas de la sociedad contemporánea ${ }^{19}$. Lo que Snow fue incapaz de apreciar (como lo demuestran sus novelas) era el hecho de que las estructuras narrativas victorianas y eduardianas habían quedado ya desbordadas por la realidad del siglo xx cuarenta años atrás, aunque en la década de los cincuenta buena parte de la crítica y de los novelistas ingleses se permitieran el lujo de pasar por alto su propia historia.

19 Citado por Rabinovitz, Rubin, en The Reaction against Experiment in the English Novel, 1950-1960, Nueva York: Columbia University Press, 1967, 116. 